\title{
Prevalence of Chlamydia trachomatis infection among women in a Middle Eastern community
}

\author{
Saad Ghazal-Aswad*1, Padmanabhan Badrinath², Nawal Osman"1, \\ Samar Abdul-Khaliq ${ }^{3}$, Shirley Mc Ilvenny ${ }^{4}$ and Islam Sidky ${ }^{5}$
}

\begin{abstract}
Address: ${ }^{1}$ Department of Obstetrics and Gynaecology, Faculty of Medicine and Health Sciences, United Arab Emirates University, P.O. Box 17666 Al-Ain, United Arab Emirates, ${ }^{2}$ Southend Primary Care Trust \& University of Cambridge, Harcourt House, Harcourt Avenue, Southend on Sea, Essex, SS2 6HE, UK, ${ }^{3}$ Serology Laboratory, Tawam Hospital, Al-Ain, United Arab Emirates, ${ }^{4}$ Department of Family \& Community Health, Faculty of Medicine, Sultan Qaboos University, Muscat, Oman and ${ }^{5}$ Department of Obstetrics \& Gynaecology, Al-Cornich Hospital, Abu Dhabi, United Arab Emirates

Email: Saad Ghazal-Aswad* - saad.ghazal_aswad@uaeu.ac.ae; Padmanabhan Badrinath - p.badrinath@southend-pct.nhs.uk; Nawal Osman - Nawal.Osman@uaeu.ac.ae; Samar Abdul-Khaliq - samarma@hotmail.com; Shirley Mc Ilvenny - mcilvenny@squ.edu.om; Islam Sidky - islamsidky@hotmail.com

* Corresponding author
\end{abstract}

Published: 27 May 2004

BMC Women's Health 2004, 4:3
Received: 05 January 2004

Accepted: 27 May 2004

This article is available from: http://www.biomedcentral.com/I472-6874/4/3

(C) 2004 Ghazal-Aswad et al; licensee BioMed Central Ltd. This is an Open Access article: verbatim copying and redistribution of this article are permitted in all media for any purpose, provided this notice is preserved along with the article's original URL.

\begin{abstract}
Background: Common vaginal infections that manifest in women are usually easily diagnosed. However, Chlamydia infection is often asymptomatic, leading to infertility before it is detected. If it occurs in pregnancy, it could lead to significant neonatal morbidity. It may also play a role with other viral infections for e.g. Human Papilloma Virus in the development of cervical cancer. The objective of this study was to determine the prevalence of Chlamydia infection in women undergoing screening for cervical abnormalities as a part of a research project in primary and secondary care institutions in the United Arab Emirates.
\end{abstract}

Methods: In this cross sectional study married women attending primary and secondary care participating in a large nationwide cervical abnormalities screening survey were offered Chlamydia testing using a commercially available test kit. This kit uses a rapid immunoassay for the direct detection of Chlamydia trachomatis antigen in endocervical swab specimens. As this study was performed in a traditional Islamic country, unmarried women were excluded from testing, as the management of any positive cases would create legal and social problems. All married women consenting to take part in the study were included irrespective of age.

Results: Of 1039 women approached over a period of eight months $919(88.5 \%)$ agreed to participate. The number of women in the 16 to 19 years was small $(0.01 \%)$ and $30 \%$ were aged over 40 years. The prevalence of Chlamydia infection in this study was $2.6 \%$ (95\% confidence interval I.2-3.3\%), which was marginally higher in women screened in secondary care $(p=0.05)$.

Conclusion: This is one of the few reports on the prevalence of Chlamydia infection in women from the Middle East. Due to cultural and social constraints this study excluded a large proportion of women aged less than 19 years of age. Hence no direct comparisons on prevalence could be made with studies from the West, which all included younger women at high risk of Chlamydia. However this study emphasizes the importance of cultural factors while interpreting results of studies from different cultures and communities. 


\section{Background}

United Arab Emirates (UAE), situated in the Arabian Gulf Peninsula, is a young country formed in 1971. Due to the judicious use of its newfound natural resources, the country has seen tremendous growth in its infrastructure including healthcare provision. According to the World Bank statistics on UAE [1], in the year 2002, the life expectancy at birth was 75.4 years, infant mortality rate 8 per 1000 and childhood immunisation rates reached 95\%. The gross national income per capita in 1998 was US $\$ 19,550$. Although economic growth has produced a dramatic improvement in the living conditions of its people, it has brought its own problems. There has been an epidemiological transition in terms of morbidity and mortality in the Emirates. According to the Ministry of Health [2], cardiovascular disorders (23.9\%), accidents $(16.8 \%)$ and cancer $(8.3 \%)$ accounted for $48.9 \%$ of the total deaths during 1998.

Although UAE is a traditional Islamic society, recent globalisation and rapid economic development have had their own impact on the health and lifestyle of the population. There are anecdotal reports of the practice of promiscuity appearing in certain sections of society. Holiday travel has also increased over the last decade in the community. Due to these reasons there is a possibility that sexually transmitted infections (STI) might pose a significant public health threat in the future. There is no populationbased data on STI in UAE. The first two authors of this report undertook a population-based study to determine the prevalence of cervical abnormalities in UAE community. As in many previous reports, $[3,4]$ this provided an ideal opportunity to establish the prevalence of Chlamydia trachomatis (CT) infection as this information is currently lacking in UAE.

CT is the most common curable sexually transmitted infection in the UK [5]. The complications of CT infection are distressing and expensive, including infertility, ectopic pregnancy, and chronic pelvic pain [6]. In most countries, selective screening, in high-risk groups, is recommended largely because testing for CT is expensive and requires considerable technical expertise. But it has been argued [7] that in high prevalence populations, selective screening should not be used and all women should be screened.

The prevalence of CT infection varies extensively depending on the health care setting. It is low in primary care but very much higher among women attending STI clinics. Ideally, to establish the prevalence in any community, screening should be undertaken at a population level. This may not always be feasible due to logistic difficulties and the delicate nature of screening for STI in healthy women. The alternative is to screen women attending primary and secondary care self-presenting themselves for a variety of reasons. This study adopted this approach and attempted to establish the prevalence of Chlamydia trachomatis infection in women in Abu Dhabi Emirate, a province of UAE to determine the magnitude of this STI in this community.

\section{Methods}

UAE is formed of seven emirates (provinces) and the largest emirate is Abu Dhabi, which covers over $3 / 4^{\text {th }}$ of the landmass where nearly $40 \%$ of the population live. The country attracts a large number of expatriates and their number is equal or in some emirates exceeds the number of UAE citizens. Due to resource constraints, only women attending primary and secondary care in Abu Dhabi Emirate were targeted for the Chlamydia prevalence survey. Both citizens and expatriates were targeted in the survey. UAE terrain is predominantly desert in nature, hence the settlements are concentrated in urban areas and a network of primary health centres serves the population. This survey was a part of a major study on prevalence of cervical abnormalities among women in UAE.

Table I: Chlamydia test refusal by socio demographic variables

\begin{tabular}{|c|c|c|c|c|c|}
\hline \multicolumn{2}{|c|}{ Characteristics } & \multicolumn{4}{|c|}{ Chlamydia Swab\# } \\
\hline & & Accepted & Refused N (\%) & $\mathrm{Chi}^{2}$ & p value \\
\hline \multirow[t]{2}{*}{ Age } & $<=$ Median & 487 & $49(9.1)$ & 6.5 & 0.011 \\
\hline & >Median & 428 & $7 I(14.2)$ & & \\
\hline \multirow[t]{2}{*}{ Education } & Illiterate & 191 & $14(6.8)$ & 2.3 & 0.133 \\
\hline & Educated & 652 & $75(10.3)$ & & \\
\hline \multirow[t]{2}{*}{ Nationality } & U.A.E. & 443 & $37(7.7)$ & 13.0 & 0.000 \\
\hline & Non-U.A.E & 468 & $82(14.9)$ & & \\
\hline \multirow[t]{2}{*}{ Gynecological complaint } & Yes & 714 & $88(11.0)$ & 1.1 & 0.284 \\
\hline & No & 198 & $31(13.5)$ & & \\
\hline
\end{tabular}

\# Figures do not always add up to 919 due to missing information 
UAE is still largely a traditional Islamic country and premarital sex is apparently very rare due to cultural, legal and societal taboos. In the only state medical school in the country there is still gender segregation and men and women medical students are taught separately. Unmarried women were excluded from testing in this study, as the management of any positive cases would create legal and social problems. Premarital sex is illegal in UAE. "According to the UAE Penal Code, he who indulges in sexual intercourse with a woman to whom he is not married even if it is with her consent, will be sentenced to no less than a year in jail" [8]. Also women attending the clinic seeking treatment specifically for vaginal discharge were excluded from the study as earlier reports show the prevalence of CT to be higher in these women $[9,10]$. Twenty-seven out of a total of 42 health centres were included in the study. These primary health centres were selected by stratified random sampling, and a ratio of $4: 1$ was adhered to for urban and rural areas i.e. for every rural centre four urban centres were selected. The strata were based on the mean monthly attendance. Chlamydia swabs were offered opportunistically to women attending gynaecology clinics during 2000 in five maternity hospitals serving the population of Abu Dhabi Emirate and the two largest major government hospitals were targeted and included in the study. An endocervical swab was taken which was analyzed using one of the commercially available kits (Clearview) according to standard protocols described by the manufacturer [11]. This is a rapid method and provides a simple direct detection assay for Chlamydia antigen in endocervical swab specimens, for either single testing or batch use. In this test Chlamydia antigen is extracted from the specimen by heating the swabs at $80^{\circ} \mathrm{C}$ with the Extraction Buffer. This detected all 15 seroversions of Chlamydia.

All positive results were reported back immediately to the health care providers who took over the responsibility of prescribing treatment and further management. UAE University medical school's research and ethics committees approved the study and administrative approval was obtained from His Excellency the Undersecretary, Ministry of Health and from the Director of Primary care, Government of UAE.

\section{Statistical analysis}

Data entry and analysis were performed using the statistical package SPSS version 10 [12]. The quality of the entry process was checked by re-entering a random sample of $10 \%$ of cases and running frequencies to check for extreme values. In the analysis, appropriate frequencies were generated. The difference between means of continuous variables was tested by "t" tests and associations between categorical variables by chi-squared and Fishers's exact test as appropriate. The prevalence of Chlamydia infection was analysed by certain sociodemographic and clinical variables.

\section{Results}

Of 1039 women approached, 919 (88.5\%) agreed to participate and the demographic characteristics of responders and non-responders are presented in Table 1. One in 4 women reported living in a polygamous marriage. Women attending primary care contributed to over $63 \%$ of the sample. The overall prevalence of vaginal Chlamydia infection among women was $2.6 \%$ and the $95 \%$ confidence interval was 1.2 to $3.3 \%$. Prevalence of infection was lower in women of polygamous marriage $(1.9 \%)$ compared to women of monogamous marriage (2.3\%) although this difference was not statistically significant. Prevalence by source of swabs, participants' age, nationality, education and presence of gynaecological complaints are presented in Table 2 .

\section{Discussion}

To our knowledge, there have been few studies on the prevalence of genital Chlamydia infection from the Middle East $[13,14]$. A hospital-based study [13] from Jordan reported a prevalence of $4.6 \%$ among symptomatic patients with urethritis using a cryptic plasmid- based PCR technique. In our study non-responders differed significantly from responders (Table 1). Contrary to a study from Sweden [15] where younger women refused participation more often than older women the reverse was true in our study. This could be due to the different cultural setting in which these studies were performed. In addition, non-UAE nationals were more likely to refuse testing. It is possible that non-nationals were concerned about being labelled as carriers of sexually transmitted infection; hence there was a higher non-response in this group. Over $60 \%$ of women had attended primary care and the prevalence of Chlamydia infection was marginally higher in women attending secondary care (Table 2). As we wanted to study the prevalence of CT in apparently healthy women, we excluded women who specifically sought care for vaginal discharge. As these women might have a higher prevalence of CT infection it is possible that their exclusion could have led to some underestimation of prevalence in this population. Although women seeking treatment for vaginal discharge were excluded, it is possible that women attending secondary care had pre-existing genital infections thus the marginally higher prevalence. The prevalence was highest in the sexually highly active age group of $16-29$ year, i.e. $16-29: 2.89 \%$ vs $30+: 1.87 \%$.

In comparing prevalence across different studies, one needs to take in to account the background risk of included women and the testing technique employed, as this would influence the prevalence rates. One consideration, which confounds the results of this survey being 
Table 2: Prevalence of Chlamydia by socio demographic characteristics, source of smear and presence of gynaecological complaints

\begin{tabular}{|c|c|c|c|}
\hline \multicolumn{2}{|c|}{ Characteristic } & \multicolumn{2}{|c|}{ Prevalence of Chlamydia } \\
\hline & & Positive N (\%) & Negative N (\%) \\
\hline \multirow[t]{2}{*}{ Source of smear* } & $\mathrm{PHC}$ & $9(1.6)$ & $565(98.4)$ \\
\hline & Hospital & $12(3.6)^{+}$ & $326(96.4)$ \\
\hline \multirow[t]{6}{*}{ Age group (years) ${ }^{\&}$} & $16-19$ & $\mathrm{I}(8.3)$ & II (9I.7) \\
\hline & $20-29$ & $8(2.7)$ & $290(97.3)$ \\
\hline & $30-39$ & $9(2.8)$ & $315(97.2)$ \\
\hline & $40-49$ & $\mathrm{I}(0.5)$ & $221(99.5)$ \\
\hline & $50-59$ & $0(0)$ & $46(100)$ \\
\hline & $>=60$ & I (16.7) & $5(83.3)$ \\
\hline \multirow[t]{6}{*}{ Nationality \| } & UAE & $10(2.3)$ & $433(97.7)$ \\
\hline & Gulf countries & $2(2.7)$ & $72(97.3)$ \\
\hline & African Arab & $3(3.4)$ & $86(96.6)$ \\
\hline & Middle East & $2(1.7)$ & $116(98.3)$ \\
\hline & Indian subcontinent & $\mathrm{I}(\mathrm{I} .0)$ & $96(99.0)$ \\
\hline & Others & $3(3.7)$ & $79(96.3)$ \\
\hline \multirow[t]{4}{*}{ Education level** } & Illiterate & $10(2.3)$ & $433(97.7)$ \\
\hline & Primary school & $2(2.7)^{\prime}$ & $72(97.3)$ \\
\hline & Secondary school & $3(3.4)$ & $86(96.6)$ \\
\hline & College \& Higher & $2(1.7)$ & $116(98.3)$ \\
\hline \multirow[t]{2}{*}{ Gynaecological Symptom ${ }^{++}$} & Positive & $16(2.26) \& \&$ & $691(97.94)$ \\
\hline & Negative & $4(2.0)$ & $194(98) 0$. \\
\hline
\end{tabular}

* Source missing in 7 subjects, ${ }^{+}$Chi2 3.7, p $=0.054$, \&Age missing in II subjects II Nationality missing in I 5 subjects, ** Education level missing in 83 subjects ${ }^{++}$Data on symptoms missing in 14 subjects, \&\&Symptoms include lower abdominal pain and vaginal discharge

compared with studies from other countries, is the age group of women included in this study. This study only included married women and excluded unmarried women aged less than 19 years and over represented women aged 40 years and over. It is likely that the population being studied is at low risk of Chlamydia. However, in UAE society premarital sex is totally forbidden and any such activity would lead to punishment by law [8] and other sanctions by society. Although no figures are available on premarital sex from this community anecdotal evidence suggests that even if it exists it is likely to be very rare. In UAE, clinicians do not often encounter young unmarried women with STI (Dr. Neriger, Consultant in Infectious disease, Tawam hospital, Al-Ain, UAE, personal communication).

The Chlamydia prevalence in this study of $2.6 \%$ was similar to studies performed in primary care in Sweden $(2.7 \%)[15]$ and Greece (3.2\%) [16] but was much lower than studies performed in other populations; Pennsylvania family planning clinics (11.2\%) [17], antenatal clinics in Bangkok (8.6\%) [18], pregnant women attending secondary care in India (17\%) [19], and in a US college gynaecology clinic (8.1\%) [20]. As described above the lower prevalence in our study could be due to the large number of low risk women included and exclusion of younger women who could potentially be at a higher risk of STI.

The prevalence of polygamy was much higher in UAE (26\%) as compared to neighbouring Kuwait [21] $(12.5 \%)$. It is possible that the lower prevalence of infection in women of polygamous marriages could be due to their husbands having a much lower prevalence of sexually transmitted infections because they live in a stable relationship within the marriage setting. Prevalence of infection did not vary by the presence of any gynaecological complaints.

In this survey, Clearview test was employed to determine the prevalence of infection. This test is easier to perform with minimum expertise as test kits can be used off the shelf. The performance of the test varies depending on the gold standard used to compare the results. The sensitivity of the test was only $50 \%$ when amplification technologies, the AMP CT (Gen-Probe) and LCx (Abbott) assays were used as a comparison [22]. However, the sensitivity rose to $76 \%$ when a DAKO IDEIA Chlamydia test was used as the gold standard [23]. The advantages and disadvantages of using Clearview for rapid detection have been discussed elsewhere [24]. According to this decision analysis in one off testing situations, rapid tests contribute significantly to the detection and treatment of Chlamydia 
infections among women. As ours was a survey of women attending health care institutions including primary health care centres and as the resources, including expertise were limited, we used the Clearview to obtain results at point of care. In low prevalence situations, tests with low sensitivity such as Clearview may not be ideal as it has been reported that even very good tests have low positive predictive value while testing low prevalence population [25]. Hence, it is possible that the prevalence in this community might be higher and this needs further studies using other tests for Chlamydia, which have a higher sensitivity.

The US preventive task force [26] has recommended that all sexually active women aged 25 and younger and other asymptomatic women at increased risk of infection should be routinely screened. Due to the selective nature of women included in our study and the type of test employed it is hard to arrive at any definite conclusions on the prevalence of genital Chlamydia based on this study and hence to put forward any firm recommendations. However it is worth mentioning here that in our population, the prevalence of Chlamydia is much lower than other populations even though $40 \%$ were recruited from secondary care.

\section{Conclusions}

In this study performed among a conservative Middle Eastern society the acceptance of CT testing was high $(89 \%)$. The prevalence of CT infection in this population was $2.6 \%$. The prevalence was marginally higher in women attending secondary care although it was not different if women had any gynaecological complaints or not. Due to the exclusion of young women and the type of test employed no firm conclusions can be drawn on the prevalence in the population. Further studies are warranted including younger women using more sensitive tests to screen for Chlamydia. However it remains to be seen how researchers in future could overcome the legal, social and logistics constraints we faced while planning to screen young unmarried women in this traditional Islamic community. Till such studies become available we propose selective Chlamydia testing of the population based on clinical grounds. Our study also emphasises the importance of cultural factors while interpreting results of studies from different cultures and communities. Further epidemiological studies are warranted in this population to determine the correlates of Chlamydia positivity to plan preventive activities.

\section{Competing interests}

None declared.

\section{Authors' contributions}

SGA is the Principle Investigator and grant holder and was involved in planning, coordinating the research, sample collection and handling, supervising data entry and analysis, writing and editing of the manuscript. PB is the Principle co-investigator and was involved in planning the epidemiological aspects of the research, coordinating and supervising data entry and analysis, and writing and editing of the manuscript. NO supervised the sample and data collection and entering data into database and helped with the analysis of data and editing the manuscript. SAK analysed the samples and edited the manuscript. SM was involved in the planning and coordination of the research at the primary health care level, and supervised data entry and analysis and edited the manuscript. IS was involved in planning and coordination of the research at the secondary care level, data analysis, and editing the manuscript.

\section{Acknowledgements}

This study was funded by grant No: NP/99/25 from the Research Grants Committee of the Faculty of Medicine \& Health Sciences, UAE University, Al-Ain, UAE.

The authors would like to thank all the three reviewers for their constructive comments and useful suggestions and Professor F. Branicki, Chair, Department of Surgery, Faculty of Medicine \& Health Sciences, UAE University for his comments on the revised manuscript.

\section{References}

I. United Arab Emirates data profile [http://devdata.world bank.org/external/CPProfile.asp?CCODE=ARE\&PTYPE=CP]

2. Ministry of Health, Statistics, UAE, Statistics [http:// www.uae.gov.ae/moh/statistics.htm]

3. Meijer CJ, Calame JJ, de Windt EJ, Risse EK, Bleker OP, Kenemans P, Quint WG, Meddens MJ: Prevalence of Chlamydia trachomatis infection in a population of asymptomatic women in a screening program for cervical cancer. Eur J Clin Microbiol Infect Dis 1989, 8:127-30.

4. Smith JR, Murdoch J, Carrington D, Frew CE, Dougall AJ, MacKinnon $H$, Baillie D, Byford DM, Forrest CA, Davis JA: Prevalence of Chlamydia trachomatis infection in women having cervical smear tests. $B M J|99|$, 302:82-4.

5. Chlamydia trachomatis: Summary and conclusions of CMO's expert advisory group. Department of Health, London, UK 1998.

6. Clinical guidelines and standards for genital chlamydia infection. Health Education Authority, London 1997.

7. Weinstock HS, Bolan GA, Kohn R, Balladares C, Back A, Oliva G: Chlamydia trachomatis infection in women: a need for universal screening in high prevalence populations? Am J Epidemiol 1992, 135:41-7.

8. Gulf News on line edition. Ask the law [http://www.gulfnews.com/Articles/uae.asp? ArticlelD=44090]. March 15, 2002

9. Williams H, Tabrizi SN, Lee W, Kovacs GT, Garland S: Adolescence and other risk factors for Chlamydia trachomatis genitourinary infection in women in Melbourne, Australia. Sex Transm Infect 2003, 79:31-4.

10. Vishwanath S, Talwar V, Prasad R, Coyaji K, Elias CJ, de Zoysa I: Syndromic management of vaginal discharge among women in a reproductive health clinic in India. Sex Transm Infect 2000, 76:303-6.

II. CLEARVIEW for health care providers [http://www.medi cal.unipath.com/Chlamydialnstructions.cfm]

12. SPSS Base for Windows [http://www.spss.com/spss /0]

13. Awwad ZM, Al-Amarat AA, Shehabi AA: Prevalence of genital chlamydial infection in symptomatic and asymptomatic Jordanian patients. Int J Infect Dis 2003, 7:206-9. 
14. Mawajdeh SM, Al-Qutob R, Schmidt A: Measuring reproductive morbidity: a community-based approach, Jordan. Health Care Women Int 2003, 24:635-49.

15. Brannstrom M, Josefsson GB, Cederberg A, Liljestrand J: Prevalence of genital Chlamydia trachomatis infection among women in a Swedish primary health care area. Scand J Infect Dis 1992, 24:4I-6.

16. Nelson ME: Prevalence of Chlamydia trachomatis infection among women in a multiphysician primary care practice. $\mathrm{Am}$ J Prev Med 1992, 8:298-302.

17. Winter L, Goldy AS, Baer C: Prevalence and epidemiological correlates of Chalamydia trachomatis in rural and urban populations. Sex Transm Dis 1990, I 7:30-36.

18. Niamsanit S, Nunthapisud P, Limpongasanurak S: Prevalence of Chlamydia trachomatis among women attending an antenatal clinic in Bangkok. Southeast Asian J Trop Med Public Health 1998, 19:609-13.

19. Paul VK, Singh M, Gupta U, Buckshee K, Bhargava VL, Takkar D, Nag VL, Bhan MK, Deorari AK: Chlamydia Trachomatis infection among pregnant women: prevalence and parental importance. Natl Med J Ind 1999, I 2: I I-4.

20. Swinker ML, Young SA, Cleavenger RL, Neely JL, Palmer JE: Prevalence of Chlamydia trachomatis cervical infection in a college gynecology clinic: relationship to other infections and clinical features. Sex Transm Dis 1988, I 5:133-6.

21. Chaleby K: Women of polygamous marriage in an inpatient psychiatric service in Kuwait. J Nerv Ment Dis 1985, I 73:56-8.

22. Lauderdale TL, Landers L, Thorneycroft I, Chapin K: Comparison of the PACE 2 assay, two amplification assays, and Clearview EIA for detection of Chlamydia trachomatis in female endocervical and urine specimens. I Clin Microbiol 1999, 37:2223-9.

23. Woolley PD, Pumphrey J: Application of 'Clearview Chlamydia' for the rapid detection of cervical chlamydial antigen. Int J STD AIDS 1997, 8:257-8.

24. Gift TL, Pate MS, Hook EW 3rd, Kassler WJ: The rapid test paradox: when fewer cases detected lead to more cases treated: a decision analysis of tests for Chlamydia trachomatis. Sex Transm Dis 1999, 26:232-40.

25. Grimes DA, Schulz KF: Uses and abuses of screening tests. Lancet 2002, 359:88|-4.

26. Screening Chlamydial infection. US Preventive Services Task Force [http://www.ahrq.gov/clinic/uspstf/uspschlm.htm]

\section{Pre-publication history}

The pre-publication history for this paper can be accessed here:

http://www.biomedcentral.com/1472-6874/4/3/prepub
Publish with Bio Med Central and every scientist can read your work free of charge

"BioMed Central will be the most significant development for disseminating the results of biomedical research in our lifetime. "

Sir Paul Nurse, Cancer Research UK

Your research papers will be:

- available free of charge to the entire biomedical community

- peer reviewed and published immediately upon acceptance

- cited in PubMed and archived on PubMed Central

- yours - you keep the copyright
BioMedcentral 